This item was submitted to Loughborough's Research Repository by the author.

Items in Figshare are protected by copyright, with all rights reserved, unless otherwise indicated.

\title{
Translation of exhaled breath volatile analyses to sport and exercise applications
}

PLEASE CITE THE PUBLISHED VERSION

https://doi.org/10.1007/s11306-017-1266-z

\section{PUBLISHER}

(c) Springer

VERSION

AM (Accepted Manuscript)

\section{PUBLISHER STATEMENT}

This work is made available according to the conditions of the Creative Commons Attribution-NonCommercialNoDerivatives 4.0 International (CC BY-NC-ND 4.0) licence. Full details of this licence are available at: https://creativecommons.org/licenses/by-nc-nd/4.0/

\section{LICENCE}

CC BY-NC-ND 4.0

\section{REPOSITORY RECORD}

Heaney, Liam M., and Martin R. Lindley. 2019. "Translation of Exhaled Breath Volatile Analyses to Sport and Exercise Applications”. figshare. https://hdl.handle.net/2134/27704. 
Title: Translation of exhaled breath volatile analyses to sport and exercise applications

Authors: Liam M Heaney ${ }^{1} \&$ Martin R Lindley $^{2}$

\section{Author affiliations:}

${ }^{1}$ Department of Cardiovascular Sciences and NIHR Leicester Biomedical Research Centre, University of Leicester, Glenfield Hospital, Leicester, UK.

${ }^{2}$ Translational Chemical Biology Research Group, School of Sport, Exercise and Health Sciences, Loughborough University, Loughborough, UK.

\section{Corresponding author:}

Dr Liam Heaney, Department of Cardiovascular Sciences and NIHR Leicester Biomedical Research Centre, University of Leicester, Glenfield Hospital, Leicester, UK. Email: L.M.Heaney@Le.ac.uk; tel: +44 (0) 1162583041

\section{ORCID ID:}

LM Heaney: 0000-0002-8791-0167

MR Lindley: 0000-0001-7686-9421 


\begin{abstract}
Exhaled breath gases are becomingly increasingly investigated for use as non-invasive measurements for clinical diagnosis, prognosis and therapeutic monitoring. Exhaled volatile organic compounds (VOCs) in the breath, which make up the exhaled volatilome, offer a rich sample medium that provides both information to external exposures as well as endogenous metabolism. For these reasons, exhaled breath analyses can be extended further beyond disease-based investigations, and used for wider biomarker measurement purposes. The use of a rapid, non-invasive (and potentially non-physically demanding) test in an exercise and/or sporting situation may provide additional information for translation to performance sport, recreational exercise/fitness and clinical exercise health. This review intends to provide an overview into the initial exploration of exhaled VOC measurements in sport and exercise science, and understand the current limitations in knowledge and instrumentation that have restricted these methodologies in becoming common practice.
\end{abstract}

\title{
Keywords
}

Condensate; Anti-doping; Mass Spectrometry; Metabolism; VOC; Volatilome 


\section{Introduction}

Research in the field of exhaled breath volatiles is developing rapidly and is predominately focussed on disease diagnosis and prognosis (Amann et al. 2014b). The ability to identify early onset of life-threatening diseases through a subtle change in an exhaled breath profile, and collected non-invasively, has been a focus of clinical interest (e.g. Cikach et al. 2014; Bos et al. 2014). However, implementation of exhaled breath analyses can extend further beyond disease diagnosis and/or management to have potential benefit to the wider community. Preliminary research into exhaled breath in exercise-based scenarios provides alternative methodologies for translation to performance sport, recreational exercise, sports medicine and exercise for health monitoring.

Exhaled breath is predominantly comprised of a mixture of nitrogen $\left(\mathrm{N}_{2}\right)$, oxygen $\left(\mathrm{O}_{2}\right)$, carbon dioxide $\left(\mathrm{CO}_{2}\right)$, inert gases and water vapour. However, trace amounts of volatile organic compounds (VOCs) are present at parts per million $\left[>0.1 \mathrm{~g} / \mathrm{m}^{3}(\mathrm{~g}), \mathrm{ppmv}\right]$, parts per billion [1 $\mu \mathrm{g} / \mathrm{m}^{3}{ }_{\mathrm{gg}}$ to $0.1 \mathrm{~g} / \mathrm{m}^{3}(\mathrm{~g})$, ppbv] and parts per trillion $\left[<1 \mu \mathrm{g} / \mathrm{m}^{3}(\mathrm{~g})\right.$, pptv] concentrations (Miekisch et al. 2004; Amann et al. 2014a). This produces an exhaled volatilome, which refers to the large variety of VOCs that are present in exhaled breath that can be collected and analysed, predominantly by mass spectrometry (MS)-based techniques (Amann et al. 2014a; Smith et al. 2014). A recent review of volatiles measured from healthy humans reported that almost 900 compounds are measured in the exhaled volatilome, with many other VOCs present in additional mediums such as blood, urine, faeces, saliva and sweat (de Lacy Costello et al. 2014). Endogenous VOCs are produced during metabolic processes, transported in the circulatory system and enter the air in the lungs by diffusion across the alveolar membrane (van der Schee et al. 2015). Various physiological and pathophysiological processes result in alterations to metabolic pathways and, therefore, changes in volatile components are apparent in the concentration of VOCs in exhaled breath (Nakhleh et al. 2017). 
As exercise is known to drive immediate physiological responses as well as longer-term adaptations to the biological system that improve physical fitness, there is premise that these acute and/or chronic changes to metabolism could be monitored via the changes in exhaled VOCs. The implementation of these techniques into everyday sporting routines offers the potential to provide additional training support that can aid athletes in their daily activity by providing information that may only have been previously available after completion of invasive, time-consuming and/or physically-exertive tests. Thus, breath tests may offer valuable and rapid information for athletes, and their coaches, about fatigue, psychological stress (previously reported for exhaled volatiles using non-exercise interventions by Turner et al. 2013), onset of illnesses (e.g. upper respiratory tract infections) and indication of current fitness level through information of the biological phase(s) of adaptation.

Although the use of exhaled breath gases is common in exercise testing, with the measurement of exhaled $\mathrm{O}_{2}$ and $\mathrm{CO}_{2}$ to estimate $\mathrm{O}_{2}$ uptake and utilisation, the investigation of exhaled volatiles in exercise-based scenarios remains in the initial stages of research. This review intends to highlight the general procedures of exhaled gas collection for exercise, and explore key contributions to preliminary investigations performed to characterise exhaled breath volatiles in exercise and sporting contexts.

\section{Exhaled gas collection techniques}

An important consideration for exhaled breath analysis is the protocol in which the VOCs are collected, stored and subsequently analysed to ensure an accurate representation and interpretation of the exhaled profile. A major complication for the choice of sampling technique is that expired air is not uniform throughout the branching sections of the respiratory system. For example, the upper airways (pharynx, larynx, trachea and bronchi) contain dead space gases that do not include VOCs diffused from the circulation. In contrast, the lower airways 
(bronchioles and alveolar sacs) do contain VOCs that have passed into the lungs from the blood, and therefore provide a snapshot of circulating VOCs. These VOCs may be endogenous in origin, but care must be taken to ensure that a measurement is not solely a reflection of the external environment (i.e. inhaled air). Dependent on the subsequent instrumentation for analysis, exhaled VOC samples can be collected at various sections of the respiratory cycle, as mixed-exhaled (e.g. Pleil and Lindstrom 1995), alveolar (or distal) (e.g. Basanta et al. 2007) and rebreathed air (e.g. King et al. 2012). Exhaled breath collection techniques have been developed in order to selectively sample individual or collective sections of the respiratory cycle using both chemical (exhaled $\mathrm{CO}_{2}$ profile) and mechanical (full-face mask pressure profile) monitoring. Collections utilising chemical monitoring have been comprehensively described previously (Miekisch et al. 2008; Lawal et al. 2017), however, mechanical monitoring can be divided into four main components (Figure 1):

i) phase $I$ - exhalation of the anatomical dead space (gases in this phase would not typically contain any endogenous VOCs);

ii) phase $I I-$ exhaled breath contains a mixture of dead space and alveolar air;

iii) phase III - exhaled breath is a result of alveolar emptying and therefore most reflects the characteristics of air in the lower airways;

iv) phase $I V$ - exhalation ceases, inhalation begins and the cycle is repeated.

There are a number of exhaled breath collection methods that provide samples that encompass the phases of the respiratory cycle. Mixed-exhaled collections contain breath from all sections of the lungs and are most commonly collected in breath canisters or inert bags (such as Tedlar®) (Pleil and Lindstrom 1995; Mochalski et al. 2013). These devices allow for a single exhalation to be collected and measured and therefore provide a snapshot of exhaled VOCs at a solitary timepoint. Breath collection bags also allow for the sampling of multiple exhalations, 
however large volume bags must be used which are bulky and therefore lack practicality. One advantage of inert bags is the ability to collect rebreathed air by performing multiple exhalations and inhalations of the same air, reducing interference from the environment through a closed-system approach. This form of collection is considered to provide an accurate representation of VOC concentrations in the breath as compounds are permitted to reach equilibrium for concentrations in the bag and airways (Ohlsson et al. 1990), however prolonged rebreathing raises safety complications due to the increased levels of inspired $\mathrm{CO}_{2}$. These techniques offer a wide snapshot of the exhaled VOCs but are limited for the detection and measurement of low-abundant and trace VOCs. To improve on sensitivity for these low concentration VOCs, retention can be improved through the pre-concentration of VOCs using solid-phase microextraction (SPME) and adsorbent powders. SPME fibres can be placed into breath canisters or bags to extract VOCs from the exhaled gases, and exhaled gases can be passed through a stainless-steel tube containing adsorbent powders. The latter technique allows for multiple exhalations to be introduced onto the adsorbent powders, and therefore provides effective pre-concentration of low-abundant and trace analytes prior to analysis (Basanta et al. 2007). Furthermore, coupling adsorbent tubes to specially designed breath sampling devices permits specific exhaled portions of the breath to be retained. For example, the adaptive breath sampler developed by Basanta et al. (2007) uses pressure transducers, connected to a full-face mask with a non-rebreathing valve, to discard the dead space gases and draw the alveolar (distal) air from each exhalation through the adsorbent tube. This generates a concentrated VOC sample that is most reflective of the air present in the lower airways. A major drawback of this technique is that pre-filtered air is required for inhalation to eliminate masking of lowabundant VOCs by high-abundant environmental molecules. This process limits the flow of air into the mask, and therefore its potential adaptation to the increased ventilation rates seen 
during exercise-based testing ( $>200 \mathrm{~L} / \mathrm{min}$ ) is limited. Each of the techniques mentioned have specific advantages and limitations and are summarised in Table 1.

In addition to staggered VOC collection and analysis, rapid and real-time methods have been developed, and are ideally suited for translation of exhaled breath tests from the laboratory to the field. This could include pitch-side for sporting applications, or bed-side for clinical use. The most commonly employed techniques for real-time breath analyses are proton transfer reaction-MS (PTR-MS) and selected ion flow tube-MS (SIFT-MS) (Smith et al. 2014). These approaches provide immediate readback of results for measurement of highly volatile compounds at concentrations in the ppbv to pptv range without the requirement of preconcentration. Furthermore, SIFT-MS can select a single, or multiple, reactant ion(s) to reduce the complexity of ion chemistries in the ionisation source. Similarly, atmospheric chemical ionisation-MS (APCI-MS) has been developed for real-time breath analysis by the coupling of a breathing mask to a Venturi jet-pump at the inlet of the ionisation source (Heaney et al. 2016). This set-up provides measurements of an induced change in exhaled profile in the pptv range, and has been performed using a transportable compact mass spectrometer. This allows future translation into field-based analyses through housing of the instrument on a portable trolley for rapid deployment for in situ testing. As with the previously described staggered collection and analysis techniques, real-time measurements of exhaled VOCs have their advantages and drawbacks; these are summarised in Table 1.

\section{Investigations into exhaled breath volatiles and exercise}

A major application of exhaled breath VOCs in exercise and sport has been following exposure to the halomethane-rich environments surrounding swimming pools that use chlorine as a disinfectant (e.g. sodium hypochlorite). Introduction of biological matter into the swimming pool (e.g. sweat, urine etc.) causes volatile halogenated disinfectant by-products 
(DBPs) to form and be released into the atmosphere (Kim et al. 2002; Judd and Bullock 2003). The production of these chemicals has been linked to the Pool Chlorine Hypothesis which proposes that the inhalation, absorption and ingestion of DPBs may cause cumulative negative health risks (Bernard et al. 2006). For example, there have been reports of associations between childhood swimming attendance and the development of pulmonary conditions, such as asthma (Bernard et al. 2006; Andersson et al. 2015). For this reason, the uptake and elimination of DBPs in the breath has been of particular interest. Aggazzotti et al. (1995) noted a onecompartment elimination of trichloromethane (known commonly as chloroform, $\mathrm{CHCl}_{3}$ ), the most abundant of halogenated DBPs in swimming pools, in the $10 \mathrm{hrs}$ following a 45 -min intensive swimming session. Four samples were collected at each timepoint and the data reported that $\mathrm{CHCl}_{3}$ could still be detected in the breath of the swimmer at $10 \mathrm{hrs}(600 \mathrm{~min})$. However, only one sample tested positive for $\mathrm{CHCl}_{3}$ content at 135 min post-swimming, and therefore a clear interpretation of the results cannot be made. In contrast, Lindstrom et al. (1997) reported a tri-exponential elimination model of $\mathrm{CHCl}_{3}$ in two swimmers, however measurements ceased at $180 \mathrm{~min}(3 \mathrm{hrs})$ post-swimming and therefore extended time point comparisons to the previous study cannot be made. A further study extended upon these initial findings by analysing a larger cohort of 12 individuals after $1 \mathrm{hr}$ swimming (Caro and Gallego 2008). This study noted a 20 -fold increase in exhaled $\mathrm{CHCl}_{3}$ post-swimming and showed an elimination time of $240 \mathrm{~min}(6 \mathrm{hrs})$, however only three swimmers were used for the extended washout analyses. A limitation to this study was present due to the use of the BioVOC ${ }^{\text {TM }}$ sampler (Markes International Ltd., Llantrisant, UK). This device traps only the final $100 \mathrm{~mL}$ of forced expired air and therefore delivers a low concentration of exhaled VOCs to the adsorbent tube for subsequent analyses, limiting the sensitivity of sample collection. For these initial DBP washout studies, small sample sizes $(\mathrm{n} \leq 3)$ were used and single breath measurements were collected at each timepoint, therefore not allowing for natural breath-to- 
breath fluctuation of exhaled concentrations to be observed. A multiple-breath approach in a larger cohort would be beneficial to confidently characterise the development and elimination of DBPs.

In an extension of the previous work carried out on targeting DBPs following a bout of swimming, Couto et al. (2017) looked to map exhaled volatile profile changes and compare the effects measured in asthmatic and non-asthmatic elite swimmers. Although a breathomics approach using SPME and Tedlar ${ }^{\circledR}$ bags was used, and several hundreds of volatiles were characterised, the authors targeted only eight VOCs that were related to lipid peroxidation. They reported that a 1-hour pool-based training bout induced a modest reduction in exhaled concentrations of these markers, but were unable to differentiate between the asthmatic and non-asthmatic participants. These data show a global change in circulating metabolites linked to oxidative stress post-exercise that is independent of airway disease. Reduced levels of oxidative stress-related metabolites post-exercise is a finding of interest. However, as the authors correctly point out, the reduced measurement of these metabolites may be due to the increased aerobic capacity and antioxidant processes of elite athletes, or simply owing to the measurements being taken immediately post-exercise where increased minute ventilation could cause the washout and dilution of exhaled VOCs. These are important factors that must be considered and suitably controlled for in future exercise-based investigations.

Although many investigations have sought to map the changes in circulating metabolites after sport-specific and/or intense exercise protocols (Heaney et al. 2017), these investigations have been predominately performed on biofluids such as urine and plasma, with non-targeted metabolomics data from exhaled breath analyses less explored. Using a more non-targeted and expansive breathomic analysis, Decombaz et al. (2013) isolated a total of 44 VOCs that exhibited pre- to post-exercise changes. Similarly to Couto et al. (2017), this investigation included a well-described and lengthy bout of exercise, with a 1-hour cycling time-trial used 
to induce the recorded metabolic shift. These extended exercise challenges are commonplace within sport and exercise science investigations and are not excessive or unrealistic for application in competitive sport. Other investigations have utilised exercise protocols that are either not sport-specific (e.g. low-intensity, short duration), or the exercise protocol itself is poorly or not described. For example, Bikov et al. (2011) reported that shifts in exhaled VOCs showed a delay after a bout of exercise, with changes in exhaled profiles emerging from 15 min post-cessation of exercise but no change reported immediately after cessation. These data suggest that a metabolic shift is induced after the onset of recovery, however a conclusion cannot be drawn as no description of intensity for the 6-min run was provided, nor an indication into the identification of the changing metabolites. Intense or prolonged exercise challenges induce physiological changes including increased $\mathrm{O}_{2}$ consumption, changes in substrate oxidation and increased blood pressure (Chan and Burns 2013), as well as changes in blood pH (Wasserman et al. 2011) which would manipulate the chemistries of VOCs present in the circulation. These responses would not be represented in shorter, low-intensity protocols and, therefore, pose limitations for understanding exhaled volatilome responses in a competitive sport scenario.

More targeted approaches to measurement of exhaled VOCs at pre-and post-exercise timepoints have been performed. Storer et al. (2014) employed a portable SIFT-MS instrument to measure exhaled concentrations of petrochemical materials such as benzene, toluene, ethylbenzene and xylene (BTEX) when exercising in close proximity to a busy road network, an exposure that has been associated with various negative health effects (Bolden et al. 2015). In this study, a 2-fold increase of exhaled BTEX was reported post-exercise and remained elevated during recovery for a period of $20 \mathrm{~min}$. This rise was not observed in participants when exercising at a distance from the roadside, highlighting an important factor of elevated 
exposure to air pollution for sporting venues situated in areas with dense motor traffic (Rundell 2012).

Small, highly-volatile molecules have also been monitored for their response to acute and repeated bouts of low-intensity exercise. Light cycling at an intensity of $75 \mathrm{~W}$ caused a sharp rise in exhaled isoprene (from approximately 100 to $400 \mathrm{ppbv}$ ) with a gradual decrease toward resting values during the exercise period and recurrent but blunted spikes in exhaled concentrations at the onset of repeated bouts (King et al. 2009). Further work identified a peripheral (extrahepatic) source of isoprene (Koc et al. 2011) and therefore it is proposed that a store of isoprene is released from the working muscles at the onset of exercise, although a physiological purpose for this mechanism is not understood. In addition to isoprene, King et al. (2009) reported an increase in exhaled acetone which followed the onset and cessation of exercise. Concentrations in a single participant rose from approximately 750 to $1000 \mathrm{ppbv}$ during exercise, with near complete reversal during the intermediary rest period (approximately $800 \mathrm{ppbv}$ ). These findings are important for improving the understanding of acetone breath measurements for use in a clinical setting. Elevated levels of exhaled acetone have been associated with the presence of type 1 and type 2 diabetes (Wang and Wang 2013), as well as showing suitability as a marker for severity of heart failure (Marcondes-Braga et al. 2012). Therefore, for acetone breath measurements to be translated into the clinic, care must be taken to ensure the patient has not undergone physical exertion prior to sampling to avoid potential false positive results. The cycling bout at $75 \mathrm{~W}$ is deemed as low-intensity, and therefore sufficient physical exertion to alter exhaled acetone levels in diseased patients could be observed from as little as the walk to clinic. Extending and contrasting from isoprene and acetone, exhaled methane has been observed to show reduced concentrations during light exercise (Szabo et al. 2015). 
Although the application of exhaled breath measurement to high-performance sport has been limited in previous investigations, a promising use has emerged to aid in screening of athletes as part of a potential anti-doping strategy. VOCs relating to prohibited substances, such as fentanyl, propofol and tetrahydrocannabinol, have been reported in exhaled gases and warrant further investigation into metabolic by-products of banned substances that may be exhaled in volatilised form within breath gases (Berchtold et al. 2014; Trefz et al. 2017).

\section{Alternative exhaled breath analyses}

In addition to the measurement of exhaled VOCs, complimentary breath analyses can be performed on non-volatile compounds that are present in exhaled aerosols. A common workflow for this approach involves the capture, cooling and liquid collection of exhaled vapours, known as exhaled breath condensate (EBC) (Davis et al. 2012). EBC can be analysed for its overall properties (e.g. pH, Davis and Hunt 2012) and through traditional biochemical techniques such as immunosorbent assays (e.g. to measure leukotrienes and cytokines, Kharitonov and Barnes 2004), as well as analytical separation techniques including capillary electrophoresis (Kuban et al. 2012) and liquid chromatography-MS (Fernandez-Peralbo et al. 2015). EBC has gained extensive interest for the study of airway inflammatory disease (e.g. asthma, Kazani and Israel 2010), often through the analysis of compounds such as isoprostanes and leukotrienes. These are considered important intermediates of the inflammatory response (Kuban and Foret 2013) and have also been observed to increase after swimming in competitive swimmers (Morissette et al. 2016).

Exercise-based EBC investigations have centred on the study of exercise-induced respiratory conditions, with patients exhibiting exercise-induced bronchoconstriction measuring fluctuations in $\mathrm{pH}$ (Bikov et al. 2014) and cysteinyl leukotriene concentration (Bikov et al. 2010), for example. However, the application of EBC analyses to competitive 
exercise and sport scenarios is less well explored. An emphasis has been placed exerciseinduced increases in oxidative stress markers such as hydrogen peroxide $\left(\mathrm{H}_{2} \mathrm{O}_{2}\right)$ and nitrogen oxide $\left(\mathrm{NO}_{2}{ }^{-}\right)$. Araneda et al. (2012) reported that elevated $\mathrm{EBC}$ levels of $\mathrm{H}_{2} \mathrm{O}_{2}$ and $\mathrm{NO}_{2}{ }^{-}$were measured post-exercise in long-distance runners after $21.1 \mathrm{~km}$ (half-marathon) and $42.2 \mathrm{~km}$ (marathon) events. These rises were not observed in runners completing a $10 \mathrm{~km}$ event, however, a subsequent study did report elevated levels of $\mathrm{H}_{2} \mathrm{O}_{2}$ at $80 \mathrm{~min}$ post-10 $\mathrm{km}$ run (Araneda et al. 2014). A further study showed that significant elevations of $\mathrm{H}_{2} \mathrm{O}_{2}$ and $\mathrm{NO}_{2}{ }^{-}$ were also achievable through a moderate (30\% of maximal oxygen uptake) 90 -min cycling exercise (Tuesta et al. 2016). Although these studies showed statistical increases in postexercise EBC levels of pro-oxidant molecules for some exercise interventions, the results remain inconsistent and appear to be driven by data points that show extreme elevations in small sample sets $(\mathrm{n}=12-17)$, with the main cluster of post-exercise samples reporting similar levels to pre-exercise measurements. Extended experiments with increased sample sizes are required to better characterise the response of pro-oxidants in $\mathrm{EBC}$ after physical exertion. $\mathrm{EBC}$ has also been successfully demonstrated for anti- doping purposes to test for prohibited substances such as methadone (Beck et al. 2011; Hamidi et al. 2017) and meldonium (Thevis et al. 2017).

One significant advancement in the analysis of exhaled breath has been the development of extractive electrospray ionisation (EESI) which allows on-line analysis of non-volatile compounds. EESI can be performed without any prior sample preparation by mixing nebulised exhaled aerosols with an ionising spray to perform an in-source liquid-liquid extraction between colliding microdroplets (Chen et al. 2006). Although EESI has not yet been applied to sport or exercise-based interventional studies, this technique has shown potential for antidoping procedures to rapidly confirm the presence of prohibited substances including atenolol, salbutamol and cocaine (Meier et al. 2012). However, it has not been demonstrated that these 
investigatory tests exhibit the necessary sensitivity to detect these compounds in exhaled breath and therefore further research is required.

\section{Summary}

The field of exhaled breath VOC analysis is continually growing and is of increasing interest for use in clinical settings (Boots et al. 2012). The richness of the exhaled volatile profile and its translation to exercise-based scenarios has been initially explored, but has yet to be extensively applied to more sport-specific scenarios. It is apparent from the literature that the expertise in these manuscripts lie within the analytical techniques and mathematical modelling, but a distinct lack of input from sport-specific exercise physiologists, athletes and coaching staff is also clear. The exercise protocols employed in a number of investigations are of low-intensity and short duration, and do not reflect a physical exertion output that can be compared to high-performance sport or recreational exercise. Although providing interesting data into potential metabolic patterns during exercise, these data are not translatable into realworld sporting scenarios. In order to maximise the potential that exhaled VOC analysis has in exercise, increased collaborative efforts from the fields of analytical chemistry and sport and exercise science are essential. In addition, the expansion of open-source volatile compound libraries that detail VOCs that have known links to metabolic processes will help accelerate the understanding of the volatilome for exercise-based investigations. It is not overlooked that a major hurdle in applying exhaled breath analyses in sport science investigations is the access to the advanced analytical technologies, such as MS. However, as the profile of MS for bioanalytical purposes expands, there are an increasing number of researchers that realise its vast capabilities for biomolecular analyses. Perhaps the most urgent aspect to aid in translation to high-performance sport is the need to readdress gas collection techniques. Although PTRMS and SIFT-MS allow excellent on-line analysis of small, highly-volatile compounds, the 
adaptation of collection and analysis instrumentation to measure the less volatile, larger VOCs during high ventilations rates during high-intensity and prolonged exercise is paramount.

With continued efforts in technological development, as well as the collaboration between analytical and exercise scientists, the field exhaled breath VOCs for exercise-based research has the ability to uncover new analyses that provide beneficial information to athletes and their support staff. 
Table 1. Exhaled breath volatile organic compound (VOC) collection techniques and their major advantages and limitations.

\section{Off-line sampling}

\section{Technique}

Breath canisters

Inert bags (e.g. Tedlar $\left.{ }^{\circledR}\right)$

SPME

Adsorbent tubes

Major advantages

- Quick and simple collection method

- Direct injection into analytical system via syringe

- Low requirement for training and technical equipment

- Robust stainless-steel construction

- Quick and simple collection method

- Direct injection into analytical system via syringe

- Collection of rebreathed air

- Low requirement for training and technical equipment

- Low cost

- Pre-concentration of VOCs

- Reconditioned for repeated use

- Short- to medium-term storage capability

- Pre-concentration of VOCs

- Collection of VOCs from multiple exhalations

- Can be coupled to filtered air source to remove environmental interferences

- Reconditioned for repeated use

- High stability for storage (> 1 month*)

- Collection of alveolar (distal) air

\section{Major limitations}

Single breath collection

- Susceptible to leaks

- No long-term sample storage capability

- Collection of mixed-exhaled air only

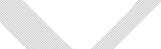

- Low stability $(<2 \mathrm{hrs})$

- Susceptible to leaks

- Susceptible to puncture

- No long-term storage capability

- Requires thermal desorption prior to injection

- Requires previous collection (e.g. inert bag)

- Pre-conditioning of single exhalation

- Requires thermal desorption prior to injection

- Requires technical training and equipment

- Different adsorbents suited to different classes of VOCs

- Initial expense relatively high

- Multiple breath collection time consuming 
On-line sampling

Technique

- Collection of alveolar (distal) air

PTR-MS

- Fully quantitative

- Low levels of detection

- Collection of alveolar (distal) air

- Selection of single or multiple reactant ion(s)

SIFT-MS

- Fully quantitative

- Capable of internal standardisation for breath humidity

- Confident identification of isobaric molecules

- Averaging of VOC response possible over multiple exhalations

APCI-MS

- Coupling to transportable MS systems

- Can be coupled to filtered air source to remove environmental interferences

- Measurements of larger, less-volatile VOCs

APCI = atmospheric pressure chemical ionisation; MS = mass spectrometry; PTR = proton transfer reaction; SIFT = selected ion flow

tube

* No changes observed in VOC profile for up to 1.5 months when stored at $-80{ }^{\circ} \mathrm{C}$ (Kang and Paul Thomas 2016) transportable purposes

- Only detects molecules with proton affinity greater than water

- Limited to measurement of highly-volatile, smaller VOCs (2)

Analysis time limited to exhalation time

- Often large and bulky apparatus not suited for transportable purposes

- Limited to measurement of highly-volatile, smaller VOCs

- Reduced limits of detection in comparison to PTR-MS

- Potential for ion sequestering in ionisation source (e.g. high concentrations of acetone in breath)

- Formation of VOC oligomers

- Quantitation requires flow of stable-isotopically labelled compounds

- Analysis time limited to exhalation time

Often large and bulky apparatus not suited for 
Figure Legends

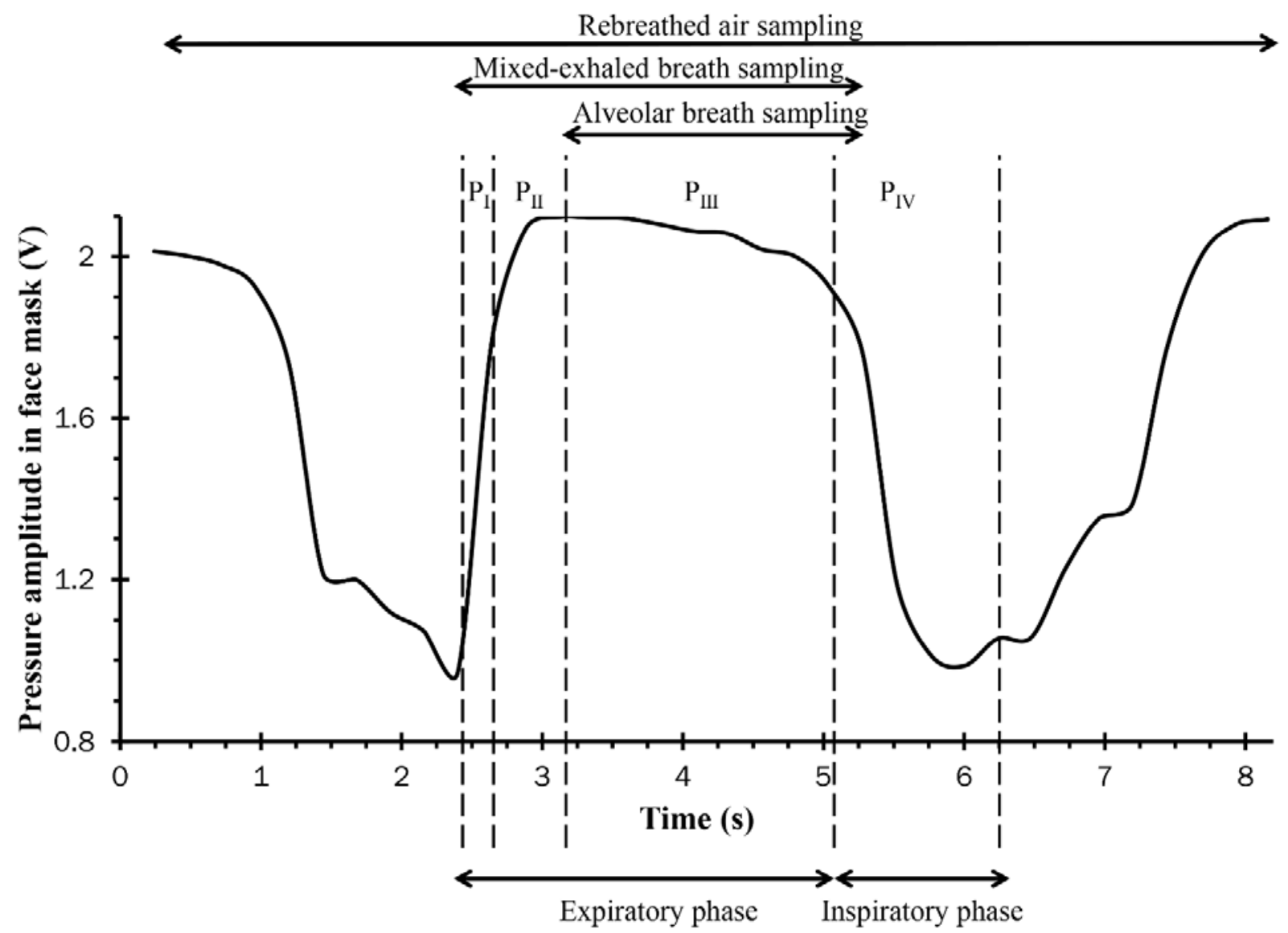

Figure 1. Example of a typical exhaled breath profile of one complete respiratory cycle illustrating the increase in exhaled gas pressure of a full-face non-rebreathing mask and detailing the fractions of rebreathed, mixed-exhaled and alveolar (distal) air used for sample capture.

Note: $\mathrm{P}_{\mathrm{I}-\mathrm{IV}}$ refer to the four phases of the respiratory cycle. 


\section{Funding}

No funding was received for the preparation of this manuscript.

\section{Conflict of Interest declaration}

The authors confirm that there are no conflicts of interest to declare.

\section{Compliance with Ethical Requirement}

No ethical requirements were present for this review article as no novel investigations generating data involving human participants are included. 


\section{References}

Aggazzotti, G., Fantuzzi, G., Righi, E., \& Predieri, G. (1995). Environmental and biological monitoring of chloroform in indoor swimming pools. J Chromatogr A, 710(1), 181-190, doi: 10.1016/00219673(95)00432-M.

Amann, A., Costello Bde, L., Miekisch, W., Schubert, J., Buszewski, B., Pleil, J., et al. (2014a). The human volatilome: volatile organic compounds (VOCs) in exhaled breath, skin emanations, urine, feces and saliva. J Breath Res, 8(3), 034001, doi:10.1088/1752-7155/8/3/034001.

Amann, A., Miekisch, W., Schubert, J., Buszewski, B., Ligor, T., Jezierski, T., et al. (2014b). Analysis of exhaled breath for disease detection. Annu Rev Anal Chem, 7, 455-482, doi:10.1146/annurevanchem-071213-020043.

Andersson, M., Hedman, L., Nordberg, G., Forsberg, B., Eriksson, K., \& Ronmark, E. (2015). Swimming pool attendance is related to asthma among atopic school children: a population-based study. Environ Health, 14, 37, doi:10.1186/s12940-015-0023-x.

Araneda, O. F., Guevara, A. J., Contreras, C., Lagos, N., \& Berral, F. J. (2012). Exhaled breath condensate analysis after long distance races. Int J Sports Med, 33(12), 955-961, doi: 10.1055/s-00321316314.

Araneda, O. F., Urbina-Stagno, R., Tuesta, M., Haichelis, D., Alvear, M., Salazar, M. P., et al. (2014). Increase of pro-oxidants with no evidence of lipid peroxidation in exhaled breath condensate after a 10 $\mathrm{km}$ race in non-athletes. J Physiol Biochem, 70(1), 107-115, doi: 10.1007/s13105-013-0285-0.

Basanta, M., Koimtzis, T., Singh, D., Wilson, I., \& Thomas, C. L. (2007). An adaptive breath sampler for use with human subjects with an impaired respiratory function. Analyst, 132(2), 153-163, doi:10.1039/b608608j.

Beck, O., Sandqvist, S., Eriksen, P., Franck, J., \& Palmskog, G. (2011). Determination of methadone in exhaled breath condensate by liquid chromatography-tandem mass spectrometry. J Anal Toxicol, 35(3), 129-133, doi: 10.1093/anatox/35.3.129.

Berchtold, C., Bosilkovska, M., Daali, Y., Walder, B., \& Zenobi, R. (2014). Real-time monitoring of exhaled drugs by mass spectrometry. Mass Spectrom Rev, 33(5), 394-413, doi:10.1002/mas.21393.

Bernard, A., Carbonnelle, S., de Burbure, C., Michel, O., \& Nickmilder, M. (2006). Chlorinated pool attendance, atopy, and the risk of asthma during childhood. Environ Health Perspect, 114(10), 1567-1573.

Bikov, A., Gajdocsi, R., Huszar, R., Szili, B., Lazar, Z., Antus, B., et al. (2010). Exercise increases exhaled breath condensate cysteinyl leukotriene concentration in asthmatic patients. J Asthma, 47(9), 1057-1062, doi: 10.1080/02770903.2010.512690.

Bikov, A., Galffy, G., Tamasi, L., Bartusek, D., Antus, B., Losonczy, G., et al. (2014). Exhaled breath condensate $\mathrm{pH}$ decreases during exercise-induced bronchoconstriction. Respirology, 19(4), 563569, doi: $10.1111 /$ resp.12248.

Bikov, A., Lazar, Z., Schandl, K., Antus, B. M., Losonczy, G., \& Horvath, I. (2011). Exercise changes volatiles in exhaled breath assessed by an electronic nose. Acta Physiol Hung, 98(3), 321-328, doi:10.1556/APhysiol.98.2011.3.9.

Bolden, A. L., Kwiatkowski, C. F., \& Colborn, T. (2015). New Look at BTEX: Are Ambient Levels a Problem? Environ Sci Technol, 49(9), 5261-5276, doi:10.1021/es505316f.

Boots, A. W., van Berkel, J. J., Dallinga, J. W., Smolinska, A., Wouters, E. F., \& van Schooten, F. J. (2012). The versatile use of exhaled volatile organic compounds in human health and disease. $J$ Breath Res, 6(2), 027108, doi:10.1088/1752-7155/6/2/027108.

Bos, L. D., Weda, H., Wang, Y., Knobel, H. H., Nijsen, T. M., Vink, T. J., et al. (2014). Exhaled breath metabolomics as a noninvasive diagnostic tool for acute respiratory distress syndrome. Eur Respir J, 44(1), 188-197, doi:10.1183/09031936.00005614. 
Caro, J., \& Gallego, M. (2008). Alveolar air and urine analyses as biomarkers of exposure to trihalomethanes in an indoor swimming pool. Environ Sci Technol, 42(13), 5002-5007.

Chan, H. H., \& Burns, S. F. (2013). Oxygen consumption, substrate oxidation, and blood pressure following sprint interval exercise. Appl Physiol Nutr Metab, 38(2), 182-187, doi:10.1139/apnm-2012-0136.

Chen, H., Venter, A., \& Cooks, R. G. (2006). Extractive electrospray ionization for direct analysis of undiluted urine, milk and other complex mixtures without sample preparation. Chem Commun, O(19), 2042-2044, doi: 10.1039/B602614A.

Cikach, F. S., Jr., Tonelli, A. R., Barnes, J., Paschke, K., Newman, J., Grove, D., et al. (2014). Breath analysis in pulmonary arterial hypertension. Chest, 145(3), 551-558, doi:10.1378/chest.13-1363.

Couto, M., Barbosa, C., Silva, D., Rudnitskaya, A., Delgado, L., Moreira, A., et al. (2017). Oxidative stress in asthmatic and non-asthmatic adolescent swimmers - a breathomics approach. Pediatr Allergy Immunol, 28(5), 452-457, doi:10.1111/pai.12729.

Davis, M. D., \& Hunt, J. (2012). Exhaled breath condensate pH assays. Immunol Allergy Clin North Am, 32(3), 377-386, doi: 10.1016/j.iac.2012.06.003.

Davis, M. D., Montpetit, A., \& Hunt, J. (2012). Exhaled breath condensate: an overview. Immunol Allergy Clin North Am, 32(3), 363-375, doi: 10.1016/j.iac.2012.06.014.

Decombaz, J., Grathwohl, D., Pollien, P., Schmitt, J. A., Borrani, F., \& Lecoultre, V. (2013). Effect of shortduration lipid supplementation on fat oxidation during exercise and cycling performance. Appl Physiol Nutr Metab, 38(7), 766-772, doi:10.1139/apnm-2012-0459.

de Lacy Costello, B., Amann, A., Al-Kateb, H., Flynn, C., Filipiak, W., Khalid, T., et al. (2014) A review of the volatiles from the healthy human body, J Breath Res, 8(1), 014001, doi:10.1088/17527155/8/1/014001.

Fernandez-Peralbo, M.A., Calderon Santiago, M., Priego-Capote, F., \& Luque de Castro, M.D. (2015). Study of exhaled breath condensate sample preparation for metabolomics analysis by LC-MS/MS in high resolution mode. Talanta, 144, 1360-1369, doi: 10.1016/j.talanta.2015.08.010.

Hamini, S., Khoubnasabjafari, M., Ansarin, K., Jouyban-Gharamaleki, V., \& Jouyban, A. (2017). Chiral separation of methadone in exhaled breath condensate using capillary electrophoresis. Anal Methods, 9, 2342, doi: 10.1039/c7ay00110j.

Heaney, L. M., Deighton, K., \& Suzuki, T. (2017). Non-targeted metabolomics in sport and exercise science. J Sports Sci, doi:10.1080/02640414.2017.1305122.

Heaney, L. M., Ruszkiewicz, D. M., Arthur, K. L., Hadjithekli, A., Aldcroft, C., Lindley, M. R., et al. (2016). Real-time monitoring of exhaled volatiles using atmospheric pressure chemical ionization on a compact mass spectrometer. Bioanalysis, 8(13), 1325-1336, doi:10.4155/bio-2016-0045.

Judd, S. J., \& Bullock, G. (2003). The fate of chlorine and organic materials in swimming pools. Chemosphere, 51(9), 869-879, doi:10.1016/s0045-6535(03)00156-5.

Kang, S., \& Paul Thomas, C. L. (2016). How long may a breath sample be stored for at -80 degrees C? A study of the stability of volatile organic compounds trapped onto a mixed Tenax:Carbograph trap adsorbent bed from exhaled breath. I Breath Res, 10(2), 026011, doi:10.1088/17527155/10/2/026011.

Kazani, S., \& Israel, E. (2010). Exhaled breath condensates in asthma: diagnostic and therapeutic implications. J Breath Res, 4(4), 047001, doi: 10.1088/1752-7155/4/4/047001.

Kharitonov, S. A., \& Barnes, P. J. (2004). Effects of corticosteroids on noninvasive biomarkers of inflammation in asthma and chronic obstructive pulmonary disease. Proc Am Thorac Soc, 1(3), 191-199.

Kim, H., Shim, J., \& Lee, S. (2002). Formation of disinfection by-products in chlorinated swimming pool water. Chemosphere, 46(1), 123-130. 
King, J., Kupferthaler, A., Unterkofler, K., Koc, H., Teschl, S., Teschl, G., et al. (2009). Isoprene and acetone concentration profiles during exercise on an ergometer. J Breath Res, 3(2), 027006, doi:10.1088/1752-7155/3/2/027006.

King, J., Unterkofler, K., Teschl, G., Teschl, S., Mochalski, P., Koc, H., et al. (2012). A modeling-based evaluation of isothermal rebreathing for breath gas analyses of highly soluble volatile organic compounds. J Breath Res, 6(1), 016005, doi:10.1088/1752-7155/6/1/016005.

Koc, H., King, J., Teschl, G., Unterkofler, K., Teschl, S., Mochalski, P., et al. (2011). The role of mathematical modeling in VOC analysis using isoprene as a prototypic example. J Breath Res, 5(3), 037102, doi:10.1088/1752-7155/5/3/037102.

Kuban, P., Kobrin, E. G., \& Kaljurand M. (2012). Capillary electrophoresis--a new tool for ionic analysis of exhaled breath condensate. J Chromatogr A, 1267, 239-246, doi: 10.1016/j.chroma.2012.06.085.

Kuban, P., \& Foret, F. (2013). Exhaled breath condensate: determination of non-volatile compounds and their potential for clinical diagnosis and monitoring. A review. Anal Chim Acta, 805, 1-18, doi: 10.1016/j.aca.2013.07.049.

Lawal, O., Ahmed, W. M., Nijsen, T. M. E., Goodacre, R., \& Fowler, S. J. (2017). Exhaled breath analysis: a review of 'breath-taking' methods for off-line analysis. Metabolomics, 13, 110, doi:10.1007/s11306-017-1241-8.

Lindstrom, A. B., Pleil, J. D., \& Berkoff, D. C. (1997). Alveolar breath sampling and analysis to assess trihalomethane exposures during competitive swimming training. Environ Health Perspect, 105(6), 636-642.

Marcondes-Braga, F. G., Gutz, I. G. R., Batista, G. L., Saldiva, P. H. N., Ayub-Ferreira, S. M., Issa, V. S., et al. (2012). Exhaled acetone as a new biomaker of heart failure severity. Chest, 142(2), 457-466, doi:10.1378/chest.11-2892.

Meier, L., Berchtold, C., Schmid, S., \& Zenobi, R. (2012). Extractive electrospray ionization mass spectrometry-enhanced sensitivity using an ion funnel. Anal Chem, 84(4), 2076-2080, doi: 10.1021/ac203022x.

Miekisch, W., Kischkel, S., Sawacki, A., Liebau, T., Mieth, M., \& Schubert, J. K. (2008). Impact of sampling procedures on the results of breath analysis. J Breath Res, 2(2), 026007, doi: 10.1088/1752$7155 / 2 / 2 / 026007$.

Miekisch, W., Schubert, J. K., \& Noeldge-Schomburg, G. F. (2004). Diagnostic potential of breath analysis-focus on volatile organic compounds. Clin Chim Acta, 347(1-2), 25-39, doi:10.1016/j.cccn.2004.04.023.

Mochalski, P., King, J., Unterkofler, K., \& Amann, A. (2013). Stability of selected volatile breath constituents in Tedlar, Kynar and Flexfilm sampling bags. Analyst, 138(5), 1405-1418, doi:10.1039/c2an36193k.

Morissette, M. C., Murray, N., Turmel, J., Milot, J., Boulet, L. P., \& Bougault, V. (2016). Increased exhaled breath condensate 8-isoprostane after a swimming session in competitive swimmers. Eur J Sport Sci, 16(5), 569-576, doi: 10.1080/17461391.2015.1063702.

Nakhleh, M. K., Haick, H., Humbert, M., \& Cohen-Kaminsky, S. (2017). Volatolomics of breath as an emerging frontier in pulmonary arterial hypertension. Eur Respir J, 49(2), 1601897, doi:10.1183/13993003.01897-2016.

Ohlsson, J., Ralph, D. D., Mandelkorn, M. A., Babb, A. L., \& Hlastala, M. P. (1990). Accurate measurement of blood alcohol concentration with isothermal rebreathing. J Stud Alcohol, 51(1), 6-13.

Pleil, J. D., \& Lindstrom, A. B. (1995). Measurement of volatile organic compounds in exhaled breath as collected in evacuated electropolished canisters. J Chromatogr B Biomed Appl, 665(2), 271-279.

Rundell, K. W. (2012). Effect of air pollution on athlete health and performance. Br J Sports Med, 46(6), 407-412, doi:10.1136/bjsports-2011-090823.

Smith, D., Spanel, P., Herbig, J., \& Beauchamp, J. (2014). Mass spectrometry for real-time quantitative breath analysis. J Breath Res, 8(2), 027101, doi:10.1088/1752-7155/8/2/027101. 
Storer, M., Salmond, J., Dirks, K. N., Kingham, S., \& Epton, M. (2014). Mobile selected ion flow tube mass spectrometry (SIFT-MS) devices and their use for pollution exposure monitoring in breath and ambient air-pilot study. J Breath Res, 8(3), 037106, doi:10.1088/1752-7155/8/3/037106.

Szabo, A., Ruzsanyi, V., Unterkofler, K., Mohacsi, A., Tuboly, E., Boros, M., et al. (2015). Exhaled methane concentration profiles during exercise on an ergometer. J Breath Res, 9(1), 016009, doi:10.1088/1752-7155/9/1/016009.

Thevis, M., Krug, O., Geyer, H., \& Schanzer, W. (2017). Expanding analytical options in sports drug testing: mass spectrometric detection of prohibited substances in exhaled breath. Rapid Commun Mass Spectrom, 31, 1290-1296, doi: 10.1002/rcm.7903.

Trefz, P., Kamysek, S., Fuchs, P., Sukul, P., Schubert, J. K., \& Miekisch, W. (2017). Drug detection in breath: non-invasive assessment of illicit or pharmaceutical drugs. J Breath Res, 11(2), 024001, doi:10.1088/1752-7163/aa61bf.

Tuesta, M., Alvear, M., Carbonell, T., Garcia, G., Guzman-Venegas, R., \& Araneda, O. F. (2016). Effect of exercise duration on pro-oxidants and $\mathrm{pH}$ in exhaled breath condensate in humans. J Physiol Biochem, 72(2), 353-360, doi: 10.1007/s13105-016-0486-4.

Turner, M. A., Bandelow, S., Edwards, L., Patel, P., Martin, H. J., Wilson, I. D., et al. (2013). The effect of a paced auditory serial addition test (PASAT) intervention on the profile of volatile organic compounds in human breath: a pilot study. J Breath Res, 7(1), 017102, doi:10.1088/17527155/7/1/017102.

van der Schee, M. P., Paff, T., Brinkman, P., van Aalderen, W. M. C., Haarman, E. G., \& Sterk, P. J. (2015). Breathomics in lung disease. Chest, 147(1), 224-231, doi:10.1378/chest.14-0781.

Wang, Z., \& Wang, C. (2013). Is breath acetone a biomarker of diabetes? A historical review on breath acetone measurements. J Breath Res, 7(3), 037109, doi:10.1088/1752-7155/7/3/037109.

Wasserman, K., Beaver, W. L., Sun, X. G., \& Stringer, W. W. (2011). Arterial H+ regulation during exercise in humans. Respir Physiol Neurobiol, 178(2), 191-195, doi:10.1016/j.resp.2011.05.018. 